\title{
A Preliminary Investigation on Periodontal Disease and Rheumatoid Arthritis
}

$\underline{\text { X.X. DONG }}{ }^{1}$, E.K.M. LI ${ }^{2}$, L.S. TAM ${ }^{2}$, K.-Y. ZEE ${ }^{1}$, G.K.C. CHIU ${ }^{1}$, and L.J. JIN ${ }^{3},{ }^{1}$ The University of Hong Kong, Hong Kong, ${ }_{2}^{2}$ The Chinese University of Hong Kong, Hong Kong, ${ }^{3}$ The University of Hong Kong, Sai Ying Pun, Hong Kong

Objectives: The present study aimed to investigate a group of patients with rheumatoid arthritis (RA) to determine their periodontal conditions and to explore a possible association with various indicators of RA. Methods: 139 Chinese patients with RA, mean age of $53.5 \pm 11.7$ years, were recruited from a university rheumatology clinic. Of them, 15 were current smokers. Following a self-reported questionnaire survey, all subjects were assessed for periodontal and rheumatological conditions, including number of missing teeth, plaque and bleeding scores, probing pocket depths (PD) and gingival recession (Rec), as well as joint pain, swelling and tenderness, physician's global assessment, joint erosion on radiographs, rheumatoid factor (RF), C-reactive protein and erythrocyte sedimentation rate. Results: The mean number of missing teeth was $5.9 \pm 6.1$, mean sites $\%$ with plaque of $65.1 \pm 22.7$, and with bleeding of $51.4 \pm 23.0$. $80 \%$ of the patients presented with PD $\geq 5 \mathrm{~mm}$ and $52 \%$ presented with PD $\geq 6 \mathrm{~mm} .66 \%$ exhibited Rec $\geq 3 \mathrm{~mm}$. Smoking RA patients showed higher sites\% with PD $\geq 5 \mathrm{~mm}(12.9 \pm 3.4$ vs. $6.9 \pm 0.9 ; \mathrm{p}<0.05)$ and $\%$ of tooth surface with $\operatorname{Rec} \geq 3 \mathrm{~mm}$ ( $26.9 \pm 7.4$ vs. $10.9 \pm 1.6 ; \mathrm{p}<0.01)$. Joint erosion was weakly correlated with both the sites\% having $P D \geq 5 \mathrm{~mm}(r=0.22, p<0.05)$ and $\%$ of tooth surface with $\operatorname{Rec} \geq 3 \mathrm{~mm}(r=0.24, p<0.05)$. The latter was also weakly correlated with RF $(r=0.27, p<0.01)$. Conclusions: This study showed that the current group of Chinese patients with rheumatoid arthritis presented with unfavorable periodontal conditions. The data suggest that there was weak correlation between severity of periodontal destruction and joint erosion as well as serum rheumatoid factor. This study was supported by the Faculty of Dentistry, HKU and the Dept. of Medicine \& Therapeutics, CUHK.

Periodontal Research - Diagnosis / Epidemiology

The Preliminary Program for International Association for Dental Research - 20th Annual Scientific Meeting of the Southeast Asia Division and Southeast Asia Association for Dental Education - 16th Annual Scientific Meeting (Sept. 1-4 2005) 